\title{
A Method for Ranking Battlefield Damaged Equipment Repairs Based on TOPSIS
}

\author{
Dong Yu-cai , Pan Xue -qin, Wang Xiao-yan, Yao Jun-jie , Liu Jun-hong , \\ Jin Qi
}

Department of Fundamental Courses, Academy of Armored Force Engineering, Beijing, 100072, China

Keywords: TOPSIS; battlefield damaged equipment; ranking repairs.

\begin{abstract}
Battlefield damaged equipment repairs of multi-objective decision-making are sorted by the method of TOPSIS. It constructs ideal solution and negative ideal solution of ranking battlefield damaged equipment repairs, compares the proximity of equipment and the ideal solution and gets the order of battlefield damaged equipment repairs. The example is applied to explain application effect of this method. The experiment shows that the method is easy to decision-making, strong objectivity, applicability and operability, has great significance to enhance the combat effectiveness of troops.
\end{abstract}

\section{Introduction}

Battlefield repair of the equipment is very important in technical support work in wartime, which is an important factor to maintain and restore the combat effectiveness of troops. It can effectively reduce the loss rate of equipment and increase the number of equipment deployed to improve the combat effectiveness[1,2,3]. Battlefield repairs have great difficulty and some limitations because of the particular environment and conditions of wartime. Improving the ability of battlefield equipment repairs is the most effective measure and fundamental way to reduce loss rate of equipment. Countermeasures to solve this difficulty is reasonable arrangement the repair order of battlefield damaged equipment under the premise of repair power in wartime, in addition to appropriately increase the strength of repair preparation. It can improve the overall security effectiveness of equipment, and ensure the troops sustained combat and win on battlefield.

The sorting or preferred of battlefield damaged equipment repairs not only need determining scientific and rational evaluation system, but also objective and rational allocation of weights of evaluation indexes. At present, commonly used methods are AHP, fuzzy comprehensive evaluation method, multi-criteria method, value engineering, and decision-making method based on entropy weight multi-objective and so on[4,5]. These methods have their own advantages, but they have a certain degree of subjective and arbitrary on subjective level of knowledge of the importance of all indicators and making the weights are uncertainties. This paper introduces a method for ranking battlefield damaged equipment repairs based on TOPSIS. The method is a pure numerical methods 。 It can objectively reflect the relative importance of each indicator and made equipment repairs sorting is more reasonable. Finally, an example of radar equipment is introduced to show the method is scientific and reliable on battlefield damaged equipment repairs sorting.

\section{Principle of TOPSIS}

The method of TOPSIS was first proposed in 1980 by Hwang et al [6]. It is a multi-objective decision making method. Its basic principle[7-11] is that the proximity of each evaluation object and the ideal solution (optimal index) are the basis to evaluate merits of each object. It is a comprehensive evaluation by means of the ideal solution and negative ideal solution and calculates the relative closeness of programs. If a program is not only close to the ideal solution, but also away from the negative ideal solution, it is the program in the collection.

(1)Data structures 
Suppose there are $\mathrm{n}$ pending assessment objects, and each object has m evaluation indicators. The original data matrix is as follows:

$$
X=\left[\begin{array}{cccc}
x_{11} & x_{12} & \cdots & x_{1 m} \\
x_{21} & x_{22} & \cdots & x_{2 m} \\
\vdots & \vdots & \vdots & \vdots \\
x_{n 1} & x_{n 2} & \cdots & x_{n m}
\end{array}\right]_{n^{*} m}
$$

(2) Indicators have same trend

Some evaluation indicators are positive, such as efficiency index, and the other are inverse, such as cost index. The evaluation requires indicators should have the same trend. Generally, inverse index are changed into positive index. If they are absolute number indicators, transformation uses the method of countdown $1 / x$. If they are relative number indicators, transformation use the method of difference $1-x$. The transformed data matrix is still denoted as $X$.

(3) Data dimensionless

Non-dimensional data matrix is recorded as $\mathrm{Y}$

$$
Y=\left[\begin{array}{cccc}
y_{11} & y_{12} & \cdots & y_{1 m} \\
y_{21} & y_{22} & \cdots & y_{2 m} \\
\vdots & \vdots & \vdots & \vdots \\
y_{n 1} & y_{n 2} & \cdots & y_{n m}
\end{array}\right]_{n^{*} m}
$$

In which

$$
y_{i j}=\frac{x_{i j}}{\sqrt{\sum_{i=2}^{n} x_{i j}^{2}}} \quad(i=1,2, \cdots, n ; j=1,2, \cdots, m)
$$

(4) Constitutes the weighted data matrix Z .

$$
z_{i j}=w_{j} * y_{i j} \quad(i=1,2, \cdots, n ; j=1,2, \cdots, m)
$$

$w_{j}$ in the above equation is the weight of the $\mathrm{j}$-th index.

(5) Identification of ideal and negative ideal value. The ideal vector is $Z^{+}$, negative ideal vector is $Z^{-}$

$$
Z^{+}=\left(z_{1}^{+}, z_{2}^{+}, \cdots, z_{m}^{+}\right), \quad Z^{-}=\left(z_{1}^{-}, z_{2}^{-}, \cdots, z_{m}^{-}\right)
$$

In which

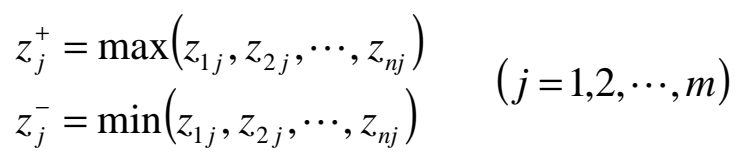


(6)Calculate the closeness between the index vector value of evaluation object and ideal vector. The distance formula uses Euclidean distance formula.

$$
\begin{aligned}
& D_{j}^{+}=\sqrt{\sum_{j=1}^{m}\left(z_{i j}-z_{j}^{+}\right)^{2}} \\
& D_{j}^{-}=\sqrt{\sum_{j=1}^{m}\left(z_{i j}-z_{j}^{+}\right)^{2}} \quad(i=1,2, \cdots, n)
\end{aligned}
$$

(7)Calculate the relative closeness between the index vector value of evaluation object and ideal vector.

$$
C_{i}=\frac{D_{i}^{-}}{D_{i}^{+}+D_{i}^{-}} \quad\left(0 \leq C_{i} \leq 1 ; i=1,2, \cdots, n\right)
$$

(8)sorting of evaluation object based on the side of closeness, $0 \leq C_{i} \leq 1$, When the indicator vector of evaluation object is ideal vector, $C_{i}=1$; When the indicator vector of evaluation object is negative ideal vector, $C_{i}=0 C_{i}$ is closer to 1 , the corresponding evaluation object is more to stand in the front.

\section{Battlefield Damaged Equipment Sorting Of Radar Network}

Information warfare increasingly dependents on intelligence information detected by early warning radar. Intelligence information is a slight delay, it will lose the best time to combat, thus it affects the process and even the outcome of wars. Therefore, the ranking of radar network battlefield damaged equipment repairs not only considers their detection efficiency ratio (ratio of detection airspace of single-radar and the radar network), necessary equipment and persons, more importantly consider the substitutability of their combat missions and repair time .

It is assumed that four radar equipments belong to different radar station in a radar network appear damage at the same time[5]. The priority of radar network battlefield damaged equipment repairs are evaluated by five indicators. The evaluation data of radar network battlefield damaged equipment determined by equipment information and battlefield damaged assessment level is shown in Table 1.There are two kinds of indicator types in the table. "1" indicates that the index is efficiency, and " 0 " indicates that the index is cost type.

Table 1 evaluation form of radar network battlefield damaged equipment repair sorting

\begin{tabular}{|c|c|c|c|c|c|c|}
\hline \multicolumn{2}{|c|}{$\begin{array}{c}\text { Battle } \\
\text { damage } \\
\text { equipment }\end{array}$} & $\begin{array}{c}\text { Substitut } \\
\text { e index of } \\
\text { combat } \\
\text { missions } x_{1}\end{array}$ & $\begin{array}{c}\text { Detection } \\
\text { efficiency } \\
\text { ratio of radar } \\
\text { network } \% x_{2}\end{array}$ & $\begin{array}{c}\text { Necessary } \\
\text { equipment of } \\
\text { repair } \\
x_{3} \text { (item) }\end{array}$ & $\begin{array}{c}\text { Necessary } \\
\text { personnel of } \\
\text { repair } \\
x_{4} \text { (person) }\end{array}$ & $\begin{array}{c}\text { Necessary } \\
\text { time of repair } \\
x_{5} \text { (hour) }\end{array}$ \\
\hline Unit & Model & 9 & 8 & 4 & 3.5 \\
\hline 1Station & LLQ $\times \times 1$ & 6 & 13 & 5 & 5 & 4 \\
\hline 3Station & LLQ $\times \times 2$ & 5 & 5 & 6 & 5 & 2.5 \\
\hline 6Station & LLQ $\times \times 3$ & 9 & 15 & 4 & 6 & 4.5 \\
\hline 7Station & LLQ $\times \times 4$ & 2 & 1 & 0 & 0 & 0 \\
\hline \multicolumn{2}{|r|}{ Indicator types } & 0 & &
\end{tabular}

Standardized indicators matrix is $\mathrm{Y}$. 


$$
Y=\left[\begin{array}{ccccc}
6 & 9 & 8 & 4 & 3.5 \\
5 & 13 & 5 & 5 & 4 \\
9 & 5 & 6 & 5 & 2.5 \\
2 & 15 & 4 & 6 & 4.5
\end{array}\right]
$$

The weighting matrix of undimensionalization indicators is $\mathrm{Z}$.

$$
Z=\left[\begin{array}{lllll}
0.2901 & 0.4025 & 0.3727 & 0.6058 & 0.4805 \\
0.3481 & 0.5814 & 0.5236 & 0.4847 & 0.4205 \\
0.1934 & 0.2236 & 0.4363 & 0.4847 & 0.6728 \\
0.8702 & 0.6708 & 0.6545 & 0.4039 & 0.3738
\end{array}\right]
$$

Ideal Solution is $Z^{+}$,

$$
Z^{+}=(0.8702,0.6708,0.6545,0.6058,0.6728)
$$

Negative ideal solution is $Z^{-}$,

$$
Z^{-}=(0.1934,0.2236,0.3272,0.4039,0.3738)
$$

The distances of evaluation object to ideal solution and sorting are shown in Table 2.

Table 2 The sorting of radar network battlefield damaged equipment

\begin{tabular}{|c|c|c|c|c|c|}
\hline \multicolumn{2}{|c|}{ Battlefield damaged equipment } & \multirow{2}{*}{$\mathrm{D}^{+}$} & \multirow{2}{*}{$\mathrm{D}^{-}$} & \multirow{2}{*}{$\mathrm{C}$} & $\begin{array}{c}\text { Sortin } \\
\mathrm{g}\end{array}$ \\
\cline { 1 - 2 } Unit & Model & & & & 3 \\
\hline 1Station & LLQ $\times \times 1$ & 0.7434 & 0.3058 & 0.2915 & 3 \\
\hline 3 Station & LLQ $\times \times 2$ & 0.6133 & 0.4463 & 0.4212 & 2 \\
\hline 6 Station & LLQ $\times \times 3$ & 0.8488 & 0.3284 & 0.2790 & 4 \\
\hline 7 Station & LLQ $\times \times 4$ & 0.3608 & 0.8748 & 0.7080 & 1 \\
\hline
\end{tabular}

If the sort uses the method of fuzzy comprehensive evaluation sorting decision-making proposed by literature [12], experts give scores to importance level of the individual indicators ,and then calculate the weight of each indicator, it has a certain degree of operability, and its results are also consistent with this article. If the sort uses the method proposed by literature [5], subjective weights are combined with objective entropy weight, the results obtained are consistent with this article. Therefore, the method is reasonable.TOPSIS is more objective to evaluation objects, sort result of battlefield damage equipment is more reasonable and scientific compared with other methods.

\section{Conclusion}

Battlefield repair is an important factor to maintain and restore combat effectiveness of equipment troops. Reasonable arrangements for the repair not only repair more damage equipments in a limit time , improve the work efficiency of the equipment repair system, but also give priority to protecting the vital equipments and improve effectiveness of battlefield repair. TOPSIS proposed by the papar can scientific sort to battlefield damaged equipment. The method is easy to decision-making, has strong objectivity, applicability and operability, and great significance to improve combat effectiveness of equipment troops .It is achieved by computer programming in practical applications. We can first establish repair database of equipment in order to improve the timeliness of command and decision in wartime . 


\section{References}

[1] Li Chuan-liang, Battlefield Repair of equipment[J]. Journal of Sichuan Ordnance, 2008 (4) :102-103.

[2] He Ji,Zhang Shi-zhong,Zhou Le-rong,etc. Study on Task Assignment Model of Wartime Equipment Maintenance[J]. Value Engineering,2010,29(35) : 295-296..

[3] Xu Lei,Wang Wen-feng,Yang Jian-jun..Tasks Assigned Model of Different Emergence Battlefield Damage [J]. CH INA SH IPREPA IR, 2008(B06): 37-39,42

[4] Yan Bing-bin, Xu Hang, Shi Quan. Decision-making Model for Ranking Battlefield Damaged Equipment Repairs Based on Multi- criteria[J]. Journal of Ordnance Engineering College. 2008, 20(5): 19-21,25.

[5] Chen Yong-jian , Guo Yi-zhong , Jiang Zhi-min,etc. A Method for Ranking Battlefield Damaged Equipment Repairs of Radar Net Based on Entropy Weight Multi-objective Decision Makin[J]. Journal of Ordnance Engineering College, 2008， 20(5): 19-21,25

[6] Friedman JH, Tukey JW.A. Projection Pursuit Algorithm for Exploratory Data Analysis[J]. IEEE Trans Compute, 1974.23(1):881-890.

[7] Zhang Lian-peng, Liu Qing-huo, Liu Guo-lin,etc. Multi-directional Projection Pursuit and Feature Extraction of Hyperspectral Remote Sensing Image[J] . Chinese Science Abstracts, 2007, 13(12): 97-97

[8] Chen Jun, Zhang Hui-sheng, Zhang Ji-guang. Performance Evaluation ofM issileW eapon Systems Based on Advanced TOPSIS Theory[ J ]. omputer Simulation2. 10,27 (9): 83-87

[9] GONG Yan-de,LI Bang-y i ,L I U Tao. Research on Comprehensive Income Coordination Strategy for Three -echelon CLSC Based on TOPSIS Method[ $\mathrm{J}$ ].Journal o f Systems \& Management, 2010.19 (3) :260-265

[10] GUO Ying-ling,LIU Hong-qi, GUO Rui-feng. A method of life cycle impact assessment based on TOPSIS[ J ].Machinery Design \& Manufacture, 2010,09:187-188

[11] ZHOU Ya. The Study of TOPSIS Method in Multiple Attribute Decision Making[D]. Wuhan University of Technology.2009.

[12] Deng Hui-yong,Ma Ji-sheng,Wang Yan,etc. Assessment of Artillery Quality Based on Fuzzy Comprehensive Evaluation[J].Journal of Gun Launch \& Control, 2008(1): 20-22. 